\title{
Calcificaciones intratesticulares en la edad pediátrica
}

\author{
Domínguez Hinarejos C, Bonillo García MA, Sangüesa C*, Serrano Durbá A, García Ibarra F.
}

Servicio de Urología Infantil. *Radiodiagnóstico. Hospital Universitario La Fe. Valencia.

Actas Urol Esp. 2007:31(1):33-37

\begin{abstract}
RESUMEN
CALCIFICACIONES INTRATESTICULARES EN LA EDAD PEDIÁTRICA

Introducción: La microlitiasis testicular (MT) constituye una entidad infrecuente en la edad pediátrica con una incidencia radiológica de 0,16 al 0,6\% según series. El amplio uso de la ecografia así como la disponibilidad de sondas de alta frecuencia ha aumentado el descubrimiento incidental de MT.

Material y Método: Desde enero de 1998 hasta octubre de 2002 se detectaron en nuestro hospital 7 niños entre 9 y 13 años (edad media 1 laños y un mes) con MT. La apariencia ecográfica de estas fue la de múltiples focos puntiformes hiperecogénicos de 1-3 mm sin sombra sónica distribuidos por el parénquima testicular.

Objetivo: Valorar los patrones ecográficos de las MT, sus asociaciones e implicaciones clínicas y determinar el manejo de las mismas.

Resultados: Todos los niños estaban asintomáticos y las motivos por las que se solicitó la ecografía fueron: criptorquidia (3), estudio por talla baja (1), estudio por ginecomastia por endocrino (1), varicocele (1) y traumatismo (1).Destacar que 3 de los casos presentaban microcalcificaciones bilaterales. De los 10 testículos con MT, 8 cumplían el criterio de MT Clásica (más de 5 microlitos/ campo) y 2 el de MT Limitada (menos de 5 microlitos/ campo). El seguimiento clínico y ecográfico está siendo anual y va desde 18 meses hasta 6 años, durante el cual no ha existido ni progresión ni regresión del número de las MT, ni tampoco en la distribución de las mismas. En ninguno de los casos ha aparecido una neoplasia testicular asociada.

Conclusiones: En revisiones amplias queda demostrado que no hay evidencia para sugerir que las MT deban ser consideradas como lesiones premalignas por sí mismas. Sin embargo la asociación MT y tumor existe. Ante esto, y debido a la falta de un protocolo consensuado, pensamos que se debe realizar tanto un seguimiento clínico (exploración manual) como ecográfico con una periodicidad anual hasta la pubertad, y posteriormente, debemos informar al paciente con el riesgo de crear un enfermo ante un hallazgo casual, de una patología incierta. No está justificado realizar pruebas más agresivas (biopsia) ante su descubrimiento, excepto: 1) si además de las MT se observa alteración en la ecogenicidad testicular y 2) si observamos cambio en el número o en la distribución de las MT en el seguimiento.
\end{abstract}

Palabras clave: Calcificaciones. Testiculares. Infancia

\section{ABSTRACT}

INTRATESTICULAR CALCIFICATIONS IN CHILDHOOD

Introduction: Testicular microlithiasis (TM) represents an uncommon occurrence at paediatrics with a radiological incidence between 0.16 to $0.6 \%$ according to reports. The greater use of ultrasound as well as the availability of high-frequency probes has increased the number of incidental TM being diagnosed.

Material and Method: From january 1998 to october 2002 seven children, aged 9 to 13 years (average 11 years and 1 months), were diagnosed of MT in our department. Sonographic appearance was hyperechogenic multiple small foci of 1-3mm without acoustic shadowing of the testicular parenchyma.

AIM: To evaluate sonographic patterns of TM, their associations, clinical consequences and to determine their management.

Results: All children were asymptomatic and the reasons for the ultrasound were: cryptorchism (3), short height (1), gynecomastia (1), varicocele (1) and scrotum trauma (1). It should be noted that 3 of the cases showed bilateral TM. Out of 10 testicles with TM, 8 met classic testicular microlithiasis (CTM) criteria (at least one image that showed five or more microliths in either or both testes) and 2 had limited testicular microlithiasis (LTM) (to have at least one microlith). Clinical and radiological follow-up is being performed annually, ranging from 18 months to 6 years, during which there hasn't been progression or regression in the number of TM or in their distribution. In none of these cases there has been shown a related testicular cancer.

Conclusions: In other broad reviews it has been shown that there is no evidence to suggest that the TM have to be considered as premalignant lesions by themselves. However, the association among TM and cancer exists. Because of that, and due to the lack of consensus, we recommend annual clinical and radiological (ultrasound) follow-up until puberty, and thereafter the patient should be informed of unknown natural history of this condition. We do not recommend more invasive procedures such as biopsy except: 1) apart form the TM there is a change in the echogenicity or 2) if there is a change in the number or distribution of the TM.

Keywords: Calcification. Testicular. Childhood. 
$\mathrm{L}^{\mathrm{a}}$ a microlitiasis testicular (MT) constituye una entidad infrecuente caracterizada ecográficamente por múltiples imágenes hiperecoicas de 2 $3 \mathrm{~mm}$ sin sombra acústica posterior, distribuidas aleatoriamente por el parénquima testicular ${ }^{1}$. Aunque fue descrita a comienzos de los años $60^{2}$ como hallazgos que representaban gonocitos disgenéticos, tras la aplicación de la microscopia electrónica se ha aportado nuevos datos sobre la etiología, la estructura y el desarrollo de los microlitos intratesticulares, sugiriendo que estos se localizan en los puntos de ruptura en la membrana basal de los túmulos seminiferos consecuencia de una obstrucción/degeneración de dichos túbulos ${ }^{3}$.

La incidencia radiológica en el adulto sintomático varía según las series de 0,16-9\% $\%^{4-6}$. El amplio uso de la ecografía en la evaluación de patologías escrotales, así como la disponibilidad de sondas de alta frecuencia, ha aumentado el diagnóstico incidental de microlitiasis a nivel testicular siendo clasificadas en clásicas o limitada en función del número de microlitos detectados en cualquier plano único ${ }^{7}$.

La historia natural de estas MT no es bien conocida en la edad pediátrica ${ }^{8}$ si bien las MT se han visto asociadas a numerosas patologias testiculares benignas, no obstante, en la población adulta también se han relacionado con tumores testiculares de células germinales hasta en un $40-45 \%$ de los $\operatorname{casos}^{7-10}$. Dada la inconsistencia existente en la literatura, la principal dificultad en su manejo radica precisamente en si una vez detectadas, deben ser consideradas como lesio- nes premalignas, y por tanto, cómo y por cuánto tiempo deben ser controladas al igual que cuál es la actitud terapéutica inicial.

Nuestro objetivo es valorar retrospectivamente los patrones ecográficos de los pacientes que presentaron MT, sus asociaciones e implicaciones clínicas y determinar el manejo de las mismas.

\section{MATERIAL Y MÉTODO}

En el periodo comprendido entre enero de 1998 y octubre 2002, se detectaron en nuestro hospital 7 niños totalmente asintomáticos con MT cuyas edades estaban comprendidas entre los 9 y 13 años (edad media 11 años y un mes). Todos los casos fueron estudiados por el mismo radiólogo con un ecógrafo Toshiba Sonolayer $\mathrm{SSH}$ 140 A con sonda lineal de 7,5MHz.

La apariencia ecográfica de estas MT fue la de múltiples focos puntiformes hiperecogénicos de 1-3mm sin sombra sónica distribuidos por el parénquima testicular. Clasificamos estos hallazgos en función del número de microlitos detectados, definiendo una MT clásica (Fig.1) si existen $>5$ microlitos / campo o limitada (Fig. 2) si es <5. Si tenemos en cuenta la distribución de estas MT, las agruparemos en focal (central/ periférica) o difusa (Fig. 3). El seguimiento clínico y ecográfico están siendo anuales y va desde 18 meses hasta 6 años.

Analizamos retrospectivamente la indicación de la ecografía escrotal, el patrón y distribución de las MT, la histología en el caso en que se realizó biopsia testicular y el seguimiento realizado en cada unos de los pacientes.
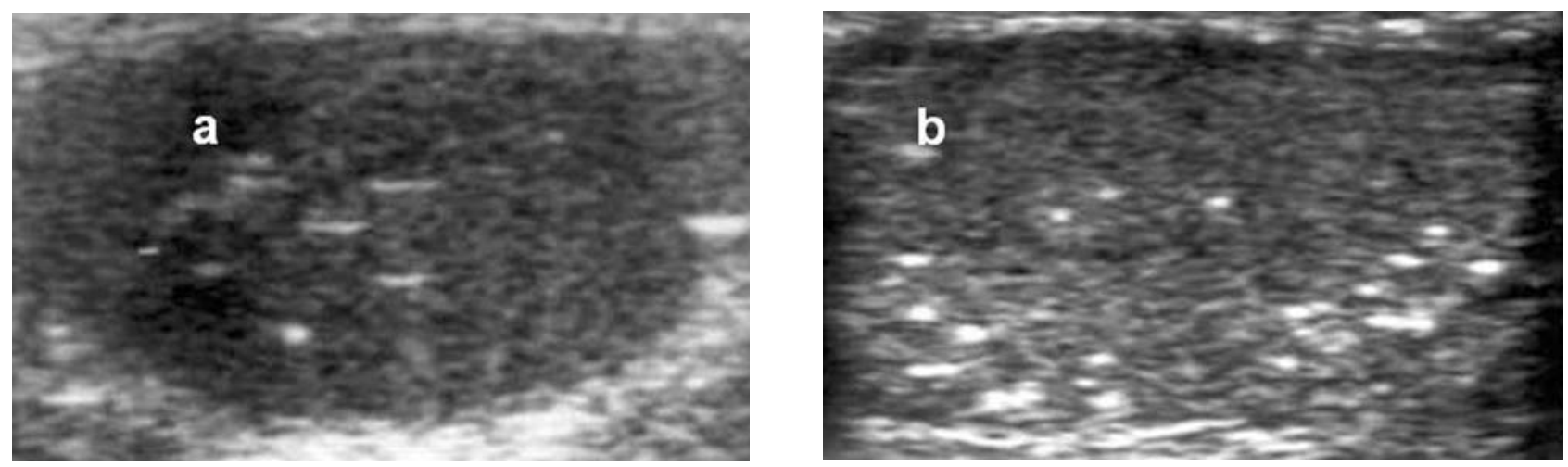

FIGURA 1. Ecografia testicular; corte longitudinal de testiculo. Microcalcificaciones testiculares clásica (MTC): focos ecogénicos puntiformes en número superior a 5 distribuidos por el parénquima testicular de forma central (a) o periférica (b). 


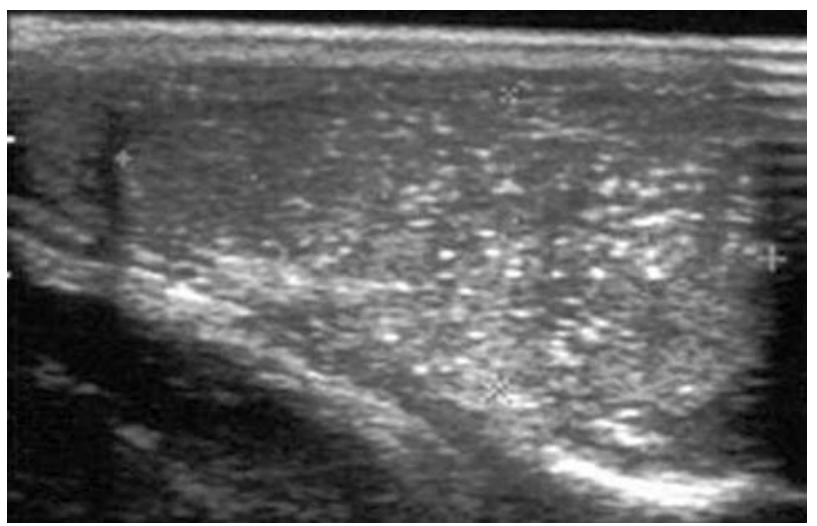

FIGURA 2. Ecografia testicular; corte longitudinal de testículo. Microcalcificaciones testiculares limitada (MTL): 2 pequeñas imágenes ecogénicas puntiformes intratesticulares.

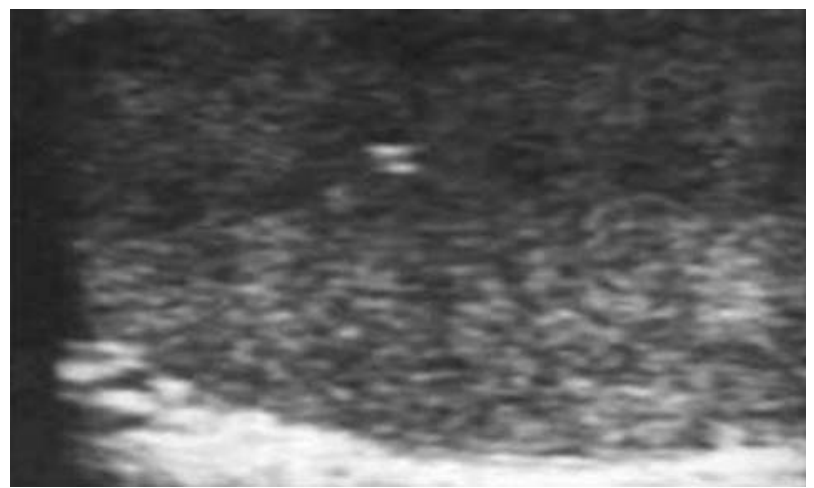

FIGURA 3. Ecografia testicular; corte longitudinal de testiculo. Microcalcificaciones testiculares clásica (MTC): focos múltiples hiperecogénicos de distribución difusa.

\section{RESULTADOS}

El motivo de consulta por el cual se solicitó una ecografia escrotal queda reflejado en la Tabla 1 , resaltando que 3 de los pacientes (43\%) presentaban una criptorquidia y que en otros 3 pacientes donde las MT eran bilaterales.

\section{Tabla 1}

Hallazgos clínico-ecográficos de los 7 pacientes estudiados en nuestro servicio por microlitiasis testicular (MT)

\begin{tabular}{lcc}
\hline Motivo de consulta & Localización & Tipo MT \\
\hline Criptorquidia & Bilateral & MTCd/Lc \\
Criptorquidia & Bilateral & MTCp \\
Varicocele & Unilateral & MTCp \\
Talla baja & Bilateral & MTCd \\
Ginecomastia & Unilateral & MTCp \\
Traumatismo & Unilateral & MTCp \\
\hline
\end{tabular}

MTC (MT Clásica). MTL (MT Limitada)

d (difusa), c (central), p (periférica)
De estos 10 testículos, 8 cumplían los criterios de MT clásica (MTC) y sólo 2 de MT limitada $(\mathrm{MTL})^{11}$. En cuanto a la distribución de los microlitos, los resultados han sido muy variables encontrándonos con los distintos patrones descritos con anterioridad.

Destacar que el primer caso visto por nosotros fue una paciente estudiado en el contexto de un talla baja que presentaba una afectación bilateral. Se le solicitó marcadores tumorales (alfa-fetoproteína y beta gonadotropina coriónica humana) que resultaron negativos, y una biopsia de ambos testes que mostró microcalcificaciones intratubulares bilaterales, maduración hasta espermatocito con focos de fibrosis tubular y túbulos con células germinales normales (Fig. 4).

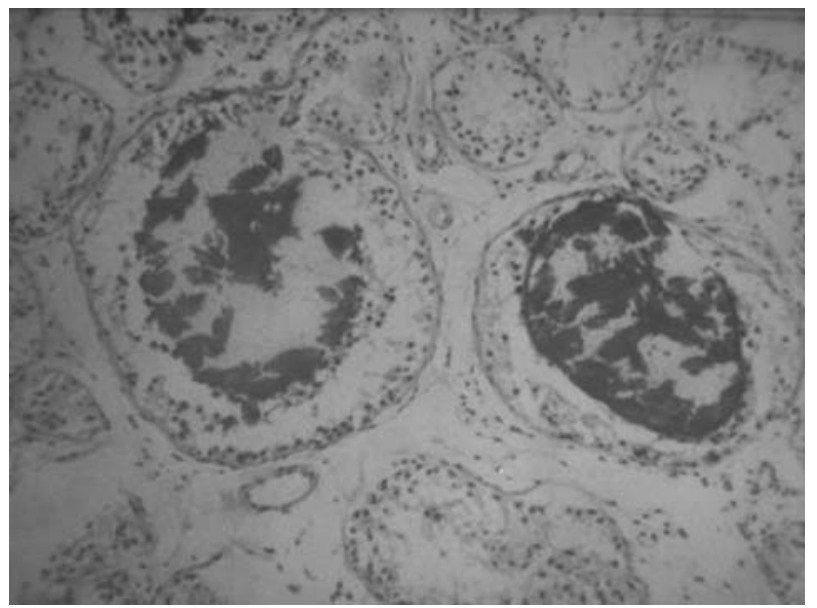

FIGURA 4: Corte histológico tras biopsia: Calcificaciones distróficas en el interior de los túbulos seminíferos.

Durante el seguimiento no ha existido ni progresión ni regresión del número de las MT, ni tampoco en la distribución de las mismas. En ninguno de los casos ha aparecido una neoplasia testicular asociada.

\section{DISCUSIÓN}

Se desconoce la incidencia real de las MT, encontrando series de autopsias de niños y adultos, donde la incidencia alcanza el 0,04\% y 4\% respectivamente ${ }^{12,13}$, mientras que en serie prospectivas de pacientes con clínica relacionada alcanza el $18,1 \%^{14}$; cabe destacar un estudio realizado en jóvenes asintomáticos donde esta prevalencia fue del $5,6 \%^{15}$. Se ha comprobado que 
las microcalcificaciones están presentes en el $74 \%$ de los pacientes con tumores testiculares malignos y sólo en el 16\% de aquellos pacientes sometidos a orquiectomías por lesiones no tumorales ${ }^{16}$

La asociación de las MT con una amplia variedad de patologías testiculares como criptorquidia $^{12,17}$, orquialgia ${ }^{18}$, torsión testicular ${ }^{19}$, torsión de hidátide ${ }^{20}$, varicocele ${ }^{21}$, infertilidad ${ }^{22}$ y tumo$\operatorname{res}^{7,10}$, apunta la posibilidad de que estas de múltiples calcificaciones sean originadas por degeneración celular en los túbulos seminífe$\operatorname{ros}^{19}$. Sin embargo, también se ha descrito casos en sindrome de Klinefelter, síndrome de Down, de Carney, enfermedad del Maullido del Gato ${ }^{23}$, fibrosis quística, microlitiasis alveolar pulmonar, calcificaciones del sistema nervioso simpático y del cerebro, por lo que se piensa que también puede tener un papel los mecanismos inmunológicos y genético ${ }^{13,21}$. Si estas asociaciones son casuales o coincidentes se desconoce. En la edad pediátrica la principal asociación ha sido con la criptorquidia y el varicocele ${ }^{12,16}$, así 3 de los niños de nuestro estudio presentaban una criptorquidia, pero llama la atención que en los 2 casos las MT eran bilaterales, tanto en el teste no descendido como en el situado en la bolsa, mientras que en el caso restante, llamativamente, las MT aparecen en el testículo que estaba en la bolsa y no el criptorquídico.

Doherty ${ }^{1}$ fue el primero en describir los hallazgos ecográficos clásicos de las MT como "innumerables ecos brillantes diminutos dispersos de forma difusa y uniforme por todas las estructuras testiculares". Posteriormente se confirmó que estos focos hiperecogénicos se limitaban al parénquima testicular y que el epidídimo y el resto del escroto estaban libres. Estas calcificaciones miden de 1-3 mm de diámetro y no presentan sombra acústica debido probablemente al pequeño tamaño que tienen. Como hemos mencionado anteriormente, las MT se han clasificado en clásicas y limitada en función del número de microlitos por campo ${ }^{24}$. Sólo 2 de los 10 testículos estudiados presentaban un forma limitada (2 y 3 microlitos/ plano respectivamente), siendo el resto de presentación clásica ( $>5$ microlitos/ plano). Bennett ${ }^{25}$ encuentra en su serie la forma limitada mucho más frecuente (62\%), pero de los
104 pacientes analizados no incluye ninguno en la edad pediátrica. En series donde se incluyen niños ${ }^{10,12,16}$ no se especifica en ningún momento el tipo de presentación. La importancia que Bennett da a la forma de presentación, detectando mayor incidencia de tumores asociados a MTC que en MTL, no es compartido por Middleton en su estudio prospectivo al no encontrar diferencias estadísticamente significativas entre los grupos y la coexistencia de tumores testiculares ${ }^{14}$.

Otro de los parámetros ecográficos que debemos destacar es la distribución de las calcificaciones en el testículo, bien si son difusas o focales (agrupadas central o periféricamente), ya que determinarán el seguimiento ecográfico posterior. Backus $^{7}$ señala que la localización de los tumores coincide con el área de mayor densidad de microlitos, y Frush ${ }^{26}$ describe la aparición de un tumor testicular en un área de agrupación de microlitos, dos años después de detectarse la mismas. Durante el seguimiento realizados a nuestros pacientes, no hemos visto ninguna variación ni en la distribución ni en el número de microlitiasis, siendo por tanto estables y no progresivas.

La existencia de neoplasias testiculares desarrolladas tras el diagnóstico de $\mathrm{MT}^{27,28}$ abre una controversia sobre si se debe considerar a esta entidad como una lesión premaligna, y sobre cuál debe ser el seguimiento de los pacientes una vez diagnosticados, y sobre la necesidad o no de realizar pruebas más agresivas, como la biopsia ${ }^{29}$. En revisiones amplias ${ }^{30-34}$ queda demostrado que no hay evidencia para sugerir que las MT deban ser consideradas como una entidad premaligna por sí misma y que representan más bien una manifestación de una disfunción testicular. La asociación de MT a condiciones que ya de por sí aumentan el riesgo de tumor testicular (criptorquidia), favorecería la hipótesis más probable acerca de su origen en la cual se considera que tanto la MT como la aparición de neoplasias sea debida aun sustrato etiológico común como podría ser la atrofia, isquemia y degeneración del tejido testicular disgenético ${ }^{13}$.

\section{CONCLUSIONES}

Si bien no consideramos que las MT como lesiones premalignas por sí mismas, la asociación MT y tumor existe, por lo que debido a la 
falta de un protocolo consensuado, pensamos que se debe realizar tanto un seguimiento clínico (exploración manual) como ecográfico con una periodicidad anual hasta la pubertad, posteriormente. Debemos informar al paciente, sin olvidar, el riesgo de crear un enfermo ante un hallazgo casual. No está justificado realizar pruebas más agresivas (biopsia) ante su descubrimiento, excepto: 1) si además de las MT se observa alteración en la ecogenicidad testicular y 2) si observamos cambio en el número o en la distribución de las microcalcificaciones en el seguimiento.

\section{REFERENCIAS}

1. Doherty FJ, Mullins TL, Sant GR, Drinkwater MA, Ucci AA, Jr. Testicular microlithiasis. A unique sonographic appearance. $J$ Ultrasound Med. 1987;6(7):389-392.

2. Bunge RG, Bradbury JT. Intratubular bodies of the human testis. J Urol. 1961;85:306-310.

3. Vegni-Talluri M, Bigliardi E, Vanni MG, Tota G. Testicular microliths: their origin and structure. J Urol. 1980;124(1): 105-107.

4. Cast JE, Nelson WM, Early AS, Biyani S, Cooksey G, Warnock NG, et al. Testicular microlithiasis: prevalence and tumor risk in a population referred for scrotal sonography. AJR Am J Roentgenol. 2000;175(6):1703-1706.

5. Hobarth K, Susani M, Szabo N, Kratzik C. Incidence of testicular microlithiasis. Urology 1992;40(5):464-467.

6. Bach AM, Hann LE, Hadar O, Shi W, Yoo HH, Giess CS, et al. Testicular microlithiasis: what is its association with testicular cancer? Radiology 2001;220(1):70-75.

7. Backus ML, Mack LA, Middleton WD, King BF, Winter TC, 3rd, True LD. Testicular microlithiasis: imaging appearances and pathologic correlation. Radiology. 1994;192(3): 781-785.

8. Dell'Acqua A, Toma P, Oddone M, Ciccone MA, Marsili E, Derchi LE. Testicular microlithiasis: US findings in six pediatric cases and literature review. Eur Radiol. 1999;9(5):940-944.

9. Miller RL, Wissman R, White S, Ragosin R. Testicular microlithiasis: a benign condition with a malignant association. $\mathrm{J}$ Clin Ultrasound. 1996;24(4):197-202.

10. Otite U, Webb JA, Oliver RT, Badenoch DF, Nargund VH. Testicular microlithiasis: is it a benign condition with malignant potential? Eur Urol. 2001;40(5):538-542.

11. Sangüesa C, Muro D, Domínguez C. Microlitiasis testiculares en la edad pediátrica. Radiologia 2002;44(7):314-318.

12. Nistal M, Paniagua R, Diez-Pardo JA. Testicular microlithiasis in 2 children with bilateral cryptorchidism. J Urol. 1979;121(4):535537.

13. Renshaw AA. Testicular calcifications: incidence, histology and proposed pathological criteria for testicular microlithiasis. J Urol.1998;160(5):1625-1628.

14. Middleton WD, Teefey SA, Santillan CS. Testicular microlithiasis: prospective analysis of prevalence and associated tumor. Radiology. 2002;224(2):425-428.

15. Peterson AC, Bauman JM, Light DE, McMann LP, Costabile RA. The prevalence of testicular microlithiasis in an asymptomatic population of men 18 to 35 years old. J Urol. 2001;166(6):20612064.

16. Furness PD, 3rd, Husmann DA, Brock JW, 3rd, Steinhardt GF, Bukowski TP, Freedman AL, et al. Multi-institutional study of testicular microlithiasis in childhood: a benign or premalignant condition? J Urol. 1998;160(3 Pt 2):1151-1154; discussion 1178.
17. Husmann DA. Cryptorchidism and its relationship to testicular neoplasia and microlithiasis. Urology. 2005;66(2): 424-426.

18. Jara Rascon J, Escribano Patino G, Herranz Amo F, Moncada Iribarren I, Hernandez Fernandez C. Testicular microlithiasis: diagnosis associated with orchialgia. Arch Esp Urol.1998;51(1): 82-85.

19. Mendez Gallart R, Rios Tallon J, Matheu Capo G, Gomez Tellado M, Montero Sanchez M, Pais Pineiro E, et al. Microlitiasis testicular. Significado clínico e implicaciones terapéuticas. Actas Urol Esp. 2000;24(10):832-835.

20. Kwan DJ, Kirsch AJ, Chang DT, Goluboff ET, Berdon WE, Hensle TW. Testicular microlithiasis in a child with torsion of the appendix testis. J Urol. 1995;153(1):183-184.

21. Ganem JP, Workman KR, Shaban SF. Testicular microlithiasis is associated with testicular pathology. Urology. 1999;53(1):209213.

22. Kessaris DN, Mellinger BC. Incidence and implication of testicular microlithiasis detected by scrotal duplex sonography in a select group of infertile men. J Urol. 1994;152(5 Pt 1):1560-1561.

23. Ortiz Gorraiz MA, Buitrago Sivianes S, Rodriguez Herrera F, Rosales Leal JL, Martinez Morcillo A, Vicente Prados FJ, et al. Testicular microlithiasis and testicular cancer. Arch Esp Urol. 2003;56(5):521-524.

24. Janzen DL, Mathieson JR, Marsh JI, Cooperberg PL, del Rio P, Golding RH, et al. Testicular microlithiasis: sonographic and clinical features. AJR Am J Roentgenol. 1992;158(5):1057-1060.

25. Bennett HF, Middleton WD, Bullock AD, Teefey SA. Testicular microlithiasis: US follow-up. Radiology. 2001; 218(2):359-363.

26. Frush DP, Kliewer MA, Madden JF. Testicular microlithiasis and subsequent development of metastatic germ cell tumor. AJR Am J Roentgenol. 1996;167(4):889-890.

27. Ringdahl E, Claybrook K, Teague JL, Northrup M. Testicular microlithiasis and its relation to testicular cancer on ultrasound findings of symptomatic men. J Urol. 2004;172(5 Pt 1):19041906.

28. Derogee M, Bevers RF, Prins HJ, Jonges TG, Elbers FH, Boon TA. Testicular microlithiasis, a premalignant condition: prevalence, histopathologic findings, and relation to testicular tumor. Urology 2001;57(6):1133-1137.

29. Skyrme RJ, Fenn NJ, Jones AR, Bowsher WG. Testicular microlithiasis in a UK population: its incidence, associations and follow-up. BJU Int 2000;86(4):482-485.

30. Zastrow S, Hakenberg OW, Wirth MP. Significance of testicular microlithiasis. Urol Int. 2005;75(1):3-7.

31. Pourbagher MA, Kilinc F, Guvel S, Pourbagher A, Egilmez T, Ozkardes H. Follow-up of testicular microlithiasis for subsequent testicular cancer development. Urol Int. 2005; 74(2):108-112; discussion 113.

32. Kocaoglu M, Bozlar U, Bulakbasi N, Saglam M, Ucoz T, Somuncu I. Testicular microlithiasis in pediatric age group: ultrasonography findings and literature review. Diagn Interv Radiol. 2005;11(1):60-65.

33. Rodriguez Villalba R, Scarfini M, Ruiz Castane E, Pomerol Monseny JM. Microlitiasis testicular: ¿existe relación con patología maligna?. Actas Urol Esp. 2004;28(1):38-39.

34. Rashid HH, Cos LR, Weinberg E, Messing EM. Testicular microlithiasis: a review and its association with testicular cancer. Urol Oncol. 2004;22(4):285-289.

Correspondencia autor: Dr. C. Domínguez Hinarejos.

Servicio de Urología. Hospital Infantil La Fe. Avda. Campanar, 21. 46009 Valencia. Tel.: 963862700

E-mail autor: dominguezhinar@hotmail.com

Información artículo: Original - Urología pediátrica

(Trabajo aceptado septiembre 2006) 